phagacytosis and never contain cellular endosymbionts she supposes that the symbiont bored its way in, like a predatory Bdellovibrio. The problem with this is that Bdellovibrio bores only through the cell wall, not the plasma membrane, and so never even enters the cell, which it also kills. Moreover, the hypothetical wall-less host would have no wall to bore through! She now favours Thermoplasma rather than mycoplasmas as potential hosts, despite the fact that Thermoplasma have branched ether-linked membrane lipids, not phospholipids, and so could not be ancestors of eukaryotes. Further, she fails to realize that the resemblances between the respiratory system of purple nonsulphur bacteria and eukaryotes could be explained non-symbiotically by the conversion of a purple non-sulphur bacterium autogenously into nucleus, cytoplasm and mitochondria, and gives only one out-ofdate reference to Mahler's extensive arguments for such an origin. Her description of which mitochondrial molecules are coded by mitochondrial DNA is inaccurate, incomplete and misleading.

The treatment of mitochondrial origins is inadequate; split-genes and RNA splicing receive only three lines and the unique mitochondrial code but half a line. The origin of the different cristal morphologies

\section{Attractive rigour}

\section{Sherrington}

The Theory of Magnetism I: Statics and Dynamics. By Daniel C. Mattis. Pp.295. ISBN 3-540-10611-1/0-387-10611-1. (Springer-Verlag: 1981.) DM 72, \$32.80.

THE study of magnetism has been one of the growth areas of the past decade. It has interest not only in its own right, but also as a testing ground for the quantummechanical theory of interacting electrons and as a convenient example for illustrating and probing important questions in statistical mechanics. Practical applications are legion, but mainly confined to ferro- and ferrimagnetism. The fundamental interest lies more in the intellectual challenge which the subject offers to pure physicists; indeed, this challenge has even attracted the attention of elementary-particle theorists.

There are two extreme approaches to the theory of magnetism, the phenomenological and the strictly fundamental. At an intermediate level lies the study of idealized models, but with wide variations in emphasis on rigour or qualitative conception. Most books on magnetism treat the basics in a fairly cavalier fashion and tend towards a semi-quantitive treatment of models. Mattis is a welcome exception. His inclinations are towards full rigour, eschewing sleights-of-hand or glossingover of difficulties. that some see as evidence for multiple symbiosis is totally ignored, as is the evolution of mechanisms to transfer nuclear gene products into mitochondria. The problem of gene transfer to the nucleus is barely mentioned.

Her classification of living organisms is better than most, but has several inconsistencies: for example, acceptance in the text of the basic distinction between Archaebacteria and Eubacteria is not reflected in the appendix. Her prokaryote phylogeny ignores the important data from 16s RNA sequences. A major defect of the book is the absence of critical phylogenetic analysis, upon which the testing of symbiotic ideas largely depends.

The book contains many errors, for example: non-Mendelian heredity in Chlamydomonas is not a consequence of uniparental transmission of chloroplasts; the gene for the ribulose biphosphate carboxylase large subunit is in the chloroplast not the nucleus; and Cyanophora has a pellicle not a cell wall. Such an important idea as the role of symbiosis in cell evolution, which Professor Margulis has done much to promote, deserves more thorough and more careful treatment than this.

T. Cavalier-Smith is a Lecturer in Biophysics at King's College, University of London.

The book is the first of a two-part set. This volume is divided almost equally between a detailed analysis of the quantummechanical basis of magnetism and an incisive study of several of the intermediate models. Subjects discussed include such modern ones as solitons, the Kondo effect, Bethe's ansatz and vortices in the $X Y$ model.

Mattis's relentless approach will not be to everyone's taste, but his book will be a useful addition to the library of anyone deeply interested in the origins of magnetism and the careful study of mathematical models. The statistical mechanician, or the particle theorist looking for hints on how to solve the lattice gauge theory problem, may, however, prefer to wait for the second volume which will cover thermodynamics and statistical mechanics.

Finally, praise must be given for the introductory chapter, 38 pages long, which spells out the history of magnetism from the earliest days to the present, places it in the perspective of the general evolution of physics and the development of Western thought, and is backed up by marvellous quotations and an impressive bibliography. This chapter can be strongly recommended to anyone interested in the history of science and, almost alone, would justify purchase of the book.

D. Sherrington is Reader in Theoretical Solid State Physics at Imperial College, University of London.

\section{Receptor development}

\author{
Eugene R. DeSombre
}

Ontogeny and Phylogeny of Hormone Receptors. By G. Csaba. Pp.172. ISBN 3-8055-2174-X. (Karger: 1981.) DM 172, $\$ 86.25$.

WhILE Professor Csaba does not entirely accomplish the awesome task of providing a synthesis and critical evaluation of our current knowledge of the ontogeny and phylogeny of hormone receptors, in this book he does provide a useful introduction to the subject. Most valuable for those who may not have closely followed Csaba's work, the monograph includes extensive data (72 figures and 18 tables) from his contributions on the interactions and biological effects of various hormones of higher organisms in Tetrahymena and Planaria. It starts from first principles on the basic concepts of cybernetics and information transfer related to hormonereceptor function and gives a general summary of our understanding of hormone-receptor interactions, paying especial attention to hormones active at the plasma membrane.

The subject of the phylogeny of receptors is presented almost exclusively using examples from studies with Tetrahymena and Planaria, with only occasional reference to other species. And while the section on ontogeny deals to a limited degree with higher animals - specifically, developmental aspects of receptors in the neonatal chick and rat - as well as with data from Tetrahymena and Planaria, neither of the two parts can be considered to be a definitive review of the topics.

Nonetheless, because of the excellent documentation of a variety of studies in these lower species, the useful introductory comments and a substantial concluding discussion, the book is more than a simple summary of Csaba's own experiments. His account of various possible developmental routes which could give rise to observed hormone and receptor patterns in different phyla is certainly valuable. Unfortunately, the rich literature on steroid hormones, the action of which is mediated through cytosolic receptors, is less well integrated into the presentation. The speculation that cytosolic receptors for steroid hormones may have evolved from membrane receptors, an interesting concept, is not well-developed in the book and the entire steroid hormone area is represented only by a few selected references.

More appropriately entitled Selected Topics on the Ontogeny and Phylogeny of Membrane Active Hormone Receptors, this monograph provides an interesting, if limited, entrée to the developmental aspects of hormone receptors.

Eugene R. DeSombre is a Professor in the Ben May Laboratory for Cancer Research and Director of the Biomedical Computation Facilities at the University of Chicago. 